\title{
Fixed Points and Stability in Neutral Stochastic Differential Equations with Variable Delays
}

\author{
Meng Wu, ${ }^{1}$ Nan-jing Huang, ${ }^{1}$ and Chang-Wen Zhao ${ }^{2}$ \\ ${ }^{1}$ Department of Mathematics, Sichuan University, Chengdu, Sichuan 610064, China \\ ${ }^{2}$ College of Business and Management, Sichuan University, Chengdu, Sichuan 610064, China
}

Correspondence should be addressed to Nan-jing Huang, nanjinghuang@hotmail.com

Received 4 April 2008; Accepted 9 June 2008

Recommended by Tomas Domínguez Benavides

We consider the mean square asymptotic stability of a generalized linear neutral stochastic differential equation with variable delays by using the fixed point theory. An asymptotic mean square stability theorem with a necessary and sufficient condition is proved, which improves and generalizes some results due to Burton, Zhang and Luo. Two examples are also given to illustrate our results.

Copyright (C) 2008 Meng Wu et al. This is an open access article distributed under the Creative Commons Attribution License, which permits unrestricted use, distribution, and reproduction in any medium, provided the original work is properly cited.

\section{Introduction}

Liapunov's direct method has been successfully used to investigate stability properties of a wide variety of differential equations. However, there are many difficulties encountered in the study of stability by means of Liapunov's direct method. Recently, Burton [1-4], Jung [5], Luo [6], and Zhang [7] studied the stability by using the fixed point theory which solved the difficulties encountered in the study of stability by means of Liapunov's direct method.

Up till now, the fixed point theory is almost used to deal with the stability for deterministic differential equations, not for stochastic differential equations. Very recently, Luo [6] studied the mean square asymptotic stability for a class of linear scalar neutral stochastic differential equations. For more details of the stability concerned with the stochastic differential equations, we refer to $[8,9]$ and the references therein.

Motivated by previous papers, in this paper, we consider the mean square asymptotic stability of a generalized linear neutral stochastic differential equation with variable delays by using the fixed point theory. An asymptotic mean square stability theorem with a necessary 
and sufficient condition is proved. Two examples is also given to illustrate our results. The results presented in this paper improve and generalize the main results in $[1,6,7]$.

\section{Main results}

Let $\left(\Omega, \mathcal{F},\left\{\mathcal{F}_{t}\right\}_{t \geq 0}, P\right)$ be a complete filtered probability space and let $W(t)$ denote a onedimensional standard Brownian motion defined on $\left(\Omega, \mathcal{F},\left\{\mathcal{F}_{t}\right\}_{t \geq 0}, P\right)$ such that $\left\{\mathcal{F}_{t}\right\}_{t \geq 0}$ is the natural filtration of $W(t)$. Let $a(t), b(t), \bar{b}(t), c(t), e(t), q(t) \in C\left(R^{+}, R\right)$, and $\tau(t), \delta(t) \in C\left(R^{+}, R^{+}\right)$ with $t-\tau(t) \rightarrow \infty$ and $t-\delta(t) \rightarrow \infty$ as $t \rightarrow \infty$. Here $C\left(S_{1}, S_{2}\right)$ denotes the set of all continuous functions $\phi: S_{1} \rightarrow S_{2}$ with the supremum norm $\|\cdot\|$.

In 2003, Burton [1] studied the equation

$$
x^{\prime}(t)=-\bar{b}(t) x(t-\tau(t))
$$

and proved the following theorem.

Theorem A (Burton [1]). Suppose that $\tau(t)=r$ and there exists a constant $\alpha<1$ such that

$$
\int_{t-r}^{t}|\bar{b}(s+r)| d s+\int_{0}^{t}|\bar{b}(s+r)| e^{-\int_{s}^{t} \bar{b}(u+r) d u} \int_{s-r}^{s}|\bar{b}(u+r)| d u d s \leq \alpha
$$

for all $t \geq 0$ and $\int_{0}^{\infty} \bar{b}(s) d s=\infty$. Then, for every continuous initial function $\phi:[-r, 0] \rightarrow \mathbb{R}$, the solution $x(t)=x(t, 0, \phi)$ of $(2.1)$ is bounded and tends to zero as $t \rightarrow \infty$.

Recently, Zhang [7] studied the generalization of (2.1) as follows:

$$
x^{\prime}(t)=-\sum_{j=1}^{n} \bar{b}_{j}(t) x\left(t-\tau_{j}(t)\right)
$$

and obtained the following theorem.

Theorem B (Zhang [7]). Suppose that $\tau_{j}$ is differential, the inverse function $g_{j}(t)$ of $t-\tau_{j}(t)$ exists, and there exists a constant $\alpha \in(0,1)$ such that for $t \geq 0, \liminf _{t \rightarrow \infty} \int_{0}^{t} Q(s) d s>-\infty$ and

$$
\begin{array}{r}
\sum_{j=1}^{n}\left[\int_{t-\tau_{j}(t)}^{t}\left|\bar{b}_{j}\left(g_{j}(s)\right)\right| d s+\int_{0}^{t} e^{-\int_{s}^{t} Q(u) d u}\left|\bar{b}_{j}(s)\right|\left|\tau_{j}^{\prime}(s)\right| d s\right. \\
\left.\quad+\int_{0}^{t} e^{-\int_{s}^{t} Q(u) d u}|Q(s)| \int_{s-\tau_{j}(s)}^{s}\left|\bar{b}_{j}\left(g_{j}(v)\right)\right| d v d s\right] \leq \alpha,
\end{array}
$$

where $Q(t)=\sum_{j=1}^{n} \bar{b}_{j}\left(g_{j}(t)\right)$. Then the zero solution of (2.3) is asymptotically stable if and only if $\int_{0}^{t} Q(s) d s \rightarrow \infty$, as $t \rightarrow \infty$.

Very recently, Luo [6] considered the following neutral stochastic differential equation:

$$
d[x(t)-q(t) x(t-\tau(t))]=[a(t) x(t)+b(t) x(t-\tau(t))] d t+[c(t) x(t)+e(t) x(t-\delta(t))] d W(t)
$$

and obtained the following theorem. 
Theorem C (Luo [6]). Let $\tau(t)$ be derivable. Assume that there exists a constant $\alpha \in(0,1)$ and a continuous function $h(t):[0, \infty) \rightarrow \mathbb{R}$ such that for $t \geq 0, \liminf _{t \rightarrow \infty} \int_{0}^{t} h(s) d s>-\infty$ and

$$
\begin{gathered}
|q(t)|+\int_{t-\tau(t)}^{t}|a(s)+h(s)| d s+\int_{0}^{t} e^{-\int_{s}^{t} h(u) d u}\left|(a(s-\tau(s))+h(s-\tau(s)))\left(1-\tau^{\prime}(s)\right)+b(s)-q(s) h(s)\right| d s \\
\quad+\int_{0}^{t} e^{-\int_{s}^{t} h(u) d u}|h(s)| \int_{s-\tau(s)}^{s}|a(u)+h(u)| d u d s+\left(\int_{0}^{t} e^{-2 \int_{s}^{t} h(u) d u}(|c(s)|+|e(s)|)^{2} d s\right)^{1 / 2} \leq \alpha .
\end{gathered}
$$

Then the zero solution of (2.5) is mean square asymptotically stable if and only if $\int_{0}^{t} h(s) d s \rightarrow \infty$, as $t \rightarrow \infty$.

Now, we consider the generalization of (2.5):

$$
d\left[x(t)-\sum_{j=1}^{n} q_{j}(t) x\left(t-\tau_{j}(t)\right)\right]=\sum_{j=1}^{n} b_{j}(t) x\left(t-\tau_{j}(t)\right) d t+\sum_{j=1}^{n} c_{j}(t) x\left(t-\delta_{j}(t)\right) d W(t)
$$

with the initial condition

$$
x(s)=\phi(s) \text { for } s \in\left[m\left(t_{0}\right), t_{0}\right]
$$

where $\phi \in C\left(\left[m\left(t_{0}\right), t_{0}\right], R\right), b_{j}(t), c_{j}(t), q_{j}(t) \in C\left(R^{+}, R\right), \tau_{j}(t), \delta_{j}(t) \in C\left(R^{+}, R^{+}\right), t-\tau_{j}(t) \rightarrow \infty$, and $t-\delta_{j}(t) \rightarrow \infty$ as $t \rightarrow \infty$ and for each $t_{0} \geq 0$,

$$
\begin{gathered}
m_{j}\left(t_{0}\right)=\min \left\{\inf \left\{s-\tau_{j}(s), s \geq t_{0}\right\}, \inf \left\{s-\delta_{j}(s), s \geq t_{0}\right\}\right\}, \\
m\left(t_{0}\right)=\min \left\{m_{j}\left(t_{0}\right), 1 \leq j \leq n\right\} .
\end{gathered}
$$

Note that (2.7) becomes (2.5) for $n=2, \tau_{1}(t)=0, \tau_{2}(t)=\tau(t), b_{1}(t)=a(t), b_{2}(t)=b(t), q_{1}(t)=$ $0, q_{2}(t)=q(t), \delta_{1}(t)=0, \delta_{2}(t)=\delta(t), c_{1}(t)=c(t)$, and $c_{2}(t)=e(t)$. Thus, we know that $(2.7)$ includes (2.1), (2.3), and (2.5) as special cases.

Our aim here is to generalize Theorems B and C to (2.7).

Theorem 2.1. Suppose that $\tau_{j}$ is differential, and there exist continuous functions $h_{j}(t):[0, \infty) \rightarrow \mathbb{R}$ for $j=1 \cdots n$ and a constant $\alpha \in(0,1)$ such that for $t \geq 0$

(i) $\liminf \operatorname{in}_{t \rightarrow \infty} \int_{0}^{t} H(s) d s>-\infty$,

(ii)

$$
\begin{aligned}
& \sum_{j=1}^{n}\left|q_{j}(t)\right|+\sum_{j=1}^{n} \int_{t-\tau_{j}(t)}^{t}\left|h_{j}(s)\right| d s+\sum_{j=1}^{n} \int_{0}^{t} e^{-\int_{s}^{t} H(u) d u} \mid\left(h_{j}\left(s-\tau_{j}(s)\right)\left(1-\tau_{j}^{\prime}(s)\right)+b_{j}(s)-q_{j}(s) H(s) \mid d s\right.
\end{aligned}
$$

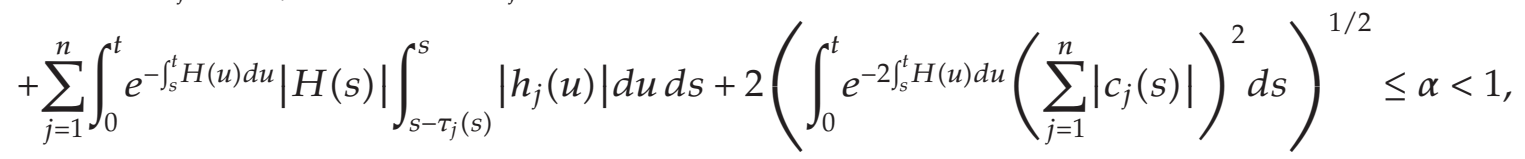

where $H(t)=\sum_{j=1}^{n} h_{j}(t)$ 
Then the zero solution of (2.7) is mean square asymptotically stable if and only if

$$
\int_{0}^{t} H(s) d s \longrightarrow \infty \text { as } t \longrightarrow \infty
$$

Proof. For each $t_{0}$, denote by $S$ the Banach space of all $\mathcal{F}$-adapted processes $\psi(t, \omega):\left[m\left(t_{0}\right), \infty\right) \times$ $\Omega \rightarrow \mathbb{R}$ which are almost surely continuous in $t$ with norm

$$
\|\psi\|_{S}=\left\{E\left(\sup _{s \geq m\left(t_{0}\right)}|\psi(s, \omega)|^{2}\right)\right\}^{1 / 2}
$$

Moreover, we set $\psi(t, \omega)=\phi(t)$ for $t \in\left[m\left(t_{0}\right), t_{0}\right]$ and $E|\psi(t, \omega)|^{2} \rightarrow 0$, as $t \rightarrow \infty$.

At first, we suppose that (2.11) holds. Define an operator $P: S \rightarrow S$ by $(P x)(t)=\phi(t)$ for $t \in\left[m\left(t_{0}\right), t_{0}\right]$ and for $t \geq t_{0}$,

$$
\begin{aligned}
(P x)(t)= & \left(\phi\left(t_{0}\right)-\sum_{j=1}^{n} q_{j}\left(t_{0}\right) \phi\left(t_{0}-\tau_{j}\left(t_{0}\right)\right)-\sum_{j=1}^{n} \int_{t_{0}-\tau_{j}\left(t_{0}\right)}^{t_{0}} h_{j}(s) \phi(s) d s\right) e^{-\int_{t_{0}}^{t} H(u) d u} \\
& +\sum_{j=1}^{n} q_{j}(t) x\left(t-\tau_{j}(t)\right)+\sum_{j=1}^{n} \int_{t-\tau_{j}(t)}^{t} h_{j}(s) x(s) d s \\
& +\int_{t_{0}}^{t} e^{-\int_{s}^{t} H(u) d u} \sum_{j=1}^{n}\left(h_{j}\left(s-\tau_{j}(s)\right)\left(1-\tau_{j}^{\prime}(s)\right)+b_{j}(s)-q_{j}(s) H(s)\right) x\left(s-\tau_{j}(s)\right) d s \\
& -\int_{t_{0}}^{t} e^{-\int_{s}^{t} H(u) d u} H(s)\left(\sum_{j=1}^{n} \int_{s-\tau_{j}(s)}^{s} h_{j}(u) x(u) d u\right) d s \\
& +\int_{t_{0}}^{t} e^{-\int_{s}^{t} H(u) d u}\left(\sum_{j=1}^{n} c_{j}(s) x\left(s-\delta_{j}(s)\right)\right) d W(s):=\sum_{i=1}^{5} I_{i}(t) .
\end{aligned}
$$

Now, we show the mean square continuity of $P$ on $\left[t_{0}, \infty\right)$. Let $x \in S, T_{1}>0$, and let $|r|$ be sufficiently small. Then

$$
E\left|(P x)\left(T_{1}+r\right)-(P x)\left(T_{1}\right)\right|^{2} \leq 5 \sum_{i=1}^{5} E\left|I_{i}\left(T_{1}+r\right)-I_{i}\left(T_{1}\right)\right|^{2} .
$$

It is easy to verify that

$$
E\left|I_{i}\left(T_{1}+r\right)-I_{i}\left(T_{1}\right)\right|^{2} \longrightarrow 0, \quad \text { as } r \longrightarrow 0, i=1,2,3,4
$$


It follows from the last term $I_{5}$ in (2.13) that

$$
\begin{aligned}
E\left|I_{5}\left(T_{1}+r\right)-I_{5}\left(T_{1}\right)\right|^{2}= & E \mid \int_{t_{0}}^{T_{1}} e^{-\int_{s}^{T_{1}} H(u) d u}\left(e^{-\int_{T_{1}}^{T_{1}+r} H(u) d u}-1\right) \sum_{j=1}^{n} c_{j}(s) x\left(s-\delta_{j}(s)\right) d W(s) \\
& \quad+\left.\int_{T_{1}}^{T_{1}+r} e^{-\int_{s}^{T_{1}+r} H(u) d u} \sum_{j=1}^{n} c_{j}(s) x\left(s-\delta_{j}(s)\right) d W(s)\right|^{2} \\
\leq & 2 E \int_{t_{0}}^{T_{1}} e^{-2 \int_{s}^{T_{1}} H(u) d u}\left(e^{-\int_{T_{1}}^{T_{1}+r} H(u) d u}-1\right)^{2}\left(\sum_{j=1}^{n}\left|c_{j}(s)\right| \cdot\left|x\left(s-\delta_{j}(s)\right)\right|\right)^{2} d s \\
& +2 E \int_{T_{1}}^{T_{1}+r} e^{-2 \int_{s}^{T_{1}+r} H(u) d u}\left(\sum_{j=1}^{n}\left|c_{j}(s)\right| \cdot\left|x\left(s-\delta_{j}(s)\right)\right|\right)^{2} d s \longrightarrow 0, \quad \text { as } r \longrightarrow 0 .
\end{aligned}
$$

Therefore, $P$ is mean square continuous on $\left[t_{0}, \infty\right)$.

Next, we verify that $P x \in S$. Since $E|x(t)| \rightarrow 0, t-\delta_{j}(t) \rightarrow \infty$ as $t \rightarrow \infty$, for each $\epsilon>0$, there exists a $T_{1}>t_{0}$ such that $s \geq T_{1}$ implies $E|x(s)|^{2}<\epsilon$ and $E\left|x\left(s-\delta_{j}(s)\right)\right|^{2}<\epsilon$. Thus, for $t \geq T_{1}$, the last term $I_{5}$ in (2.13) satisfies

$$
\begin{aligned}
& E\left|I_{5}(t)\right|^{2} \\
& \leq E \int_{t_{0}}^{T_{1}} e^{-2 \int_{s}^{t} H(u) d u}\left(\sum_{j=1}^{n} c_{j}(s) x\left(s-\delta_{j}(s)\right)\right)^{2} d s+E \int_{T_{1}}^{t} e^{-2 \int_{s}^{t} H(u) d u}\left(\sum_{j=1}^{n} c_{j}(s) x\left(s-\delta_{j}(s)\right)\right)^{2} d s \\
& \leq E\left(\sup _{s \geq m\left(t_{0}\right)}|x(s)|^{2}\right) \int_{t_{0}}^{T_{1}} e^{-2 \int_{s}^{t} H(u) d u}\left(\sum_{j=1}^{n}\left|c_{j}(s)\right|\right)^{2} d s+\epsilon \int_{T_{1}}^{t} e^{-2 \int_{s}^{t} H(u) d u}\left(\sum_{j=1}^{n}\left|c_{j}(s)\right|\right)^{2} d s .
\end{aligned}
$$

By condition (ii) and (2.11), there exists $T_{2}>T_{1}$ such that $t \geq T_{2}$ implies

$$
E\left|I_{5}(t)\right|^{2}<\epsilon+\alpha \epsilon .
$$

Thus, $E\left|I_{5}(t)\right|^{2} \rightarrow 0$, as $t \rightarrow \infty$. Similarly, we can show that $E\left|I_{i}(t)\right|^{2} \rightarrow 0, i=1,2,3,4$, as $t \rightarrow \infty$. Thus, $E|(P x)(t)|^{2} \rightarrow 0$ as $t \rightarrow \infty$. This yields $P x \in S$.

Now we show that $P: S \rightarrow S$ is a contraction mapping. From (ii), we can choose $\varepsilon>0$ such that $\alpha^{2}+\varepsilon<1$. Thus, for each $t_{0} \geq 0$, we can find a constant $L>0$ such that

$$
\begin{aligned}
& \left(1+\frac{1}{L}\right)\left(\sum_{j=1}^{n}\left|q_{j}(t)\right|+\sum_{j=1}^{n} \int_{t_{0}}^{t} e^{-\int_{s}^{t} H(u) d u}|H(s)| \int_{s-\tau_{j}(s)}^{s}\left|h_{j}(u)\right| d u d s\right. \\
& \quad+\sum_{j=1}^{n} \int_{t-\tau_{j}(t)}^{t}\left|h_{j}(s)\right| d s+\sum_{j=1}^{n} \int_{t_{0}}^{t} e^{-\int_{s}^{t} H(u) d u} \mid\left(h_{j}\left(s-\tau_{j}(s)\right)\left(1-\tau_{j}^{\prime}(s)\right)+b_{j}(s)-q_{j}(s) H(s) \mid d s\right)^{2} \\
& +4(1+L) \int_{t_{0}}^{t} e^{-2 \int_{s}^{t} H(u) d u}\left(\sum_{j=1}^{n}\left|c_{j}(s)\right|\right)^{2} d s \leq \alpha^{2}+\varepsilon<1 .
\end{aligned}
$$


For any $x, y \in S$, it follows from (2.13), conditions (i) and (ii), and Doob's $L^{p}$-inequality (see [10]) that

$$
\begin{aligned}
& e \sup _{s \geq m\left(t_{0}\right)}|(p x)(s)-(p y)(s)|^{2} \\
& =e \sup _{s \geq t_{0}} \mid \sum_{j=1}^{n} q_{j}(s)\left(x\left(s-\tau_{j}(s)\right)-y\left(s-\tau_{j}(s)\right)\right)+\sum_{j=1}^{n} \int_{s-\tau_{j}(s)}^{s} h_{j}(v)(x(v)-y(v)) d v \\
& +\int_{t_{0}}^{s} e^{-\int_{v}^{s} h(u) d u} \sum_{j=1}^{n}\left(h_{j}\left(v-\tau_{j}(v)\right)\left(1-\tau_{j}^{\prime}(v)\right)+b_{j}(v)-q_{j}(v) h(v)\right) \\
& \times\left(x\left(v-\tau_{j}(v)\right)-y\left(v-\tau_{j}(v)\right)\right) d v \\
& -\int_{t_{0}}^{s} e^{-\int_{v}^{s} h(u) d u} h(v)\left(\sum_{j=1}^{n} \int_{v-\tau_{j}(v)}^{v} h_{j}(u)(x(u)-y(u)) d u\right) d v \\
& +\left.\int_{t_{0}}^{s} e^{-\int_{v}^{s} h(u) d u}\left(\sum_{j=1}^{n} c_{j}(v)\left(x\left(v-\delta_{j}(v)\right)-y\left(v-\delta_{j}(v)\right)\right)\right) d w(v)\right|^{2} \\
& \leq\left(1+\frac{1}{l}\right) e \sup _{s \geq t_{0}}\left(\sum_{j=1}^{n}\left|q_{j}(s)\right| \cdot\left|x\left(s-\tau_{j}(s)\right)-y\left(s-\tau_{j}(s)\right)\right|\right. \\
& +\sum_{j=1}^{n} \int_{s-\tau_{j}(s)}^{s}\left|h_{j}(v)\right| \cdot|x(v)-y(v)| d v \\
& +\int_{t_{0}}^{s} e^{-\int_{v}^{S} h(u) d u} \sum_{j=1}^{n}\left|h_{j}\left(v-\tau_{j}(v)\right)\left(1-\tau_{j}^{\prime}(v)\right)+b_{j}(v)-q_{j}(v) h(v)\right| \\
& \cdot\left|x\left(v-\tau_{j}(v)\right)-y\left(v-\tau_{j}(v)\right)\right| d v \\
& \left.+\int_{t_{0}}^{s} e^{-\int_{v}^{s} h(u) d u} h(v)\left(\sum_{j=1}^{n} \int_{v-\tau_{j}(v)}^{v}\left|h_{j}(u)\right| \cdot|x(u)-y(u)| d u\right) d v\right)^{2} \\
& +4(1+l) \sup _{s \geq t_{0}}\left\{e \int_{t_{0}}^{s} e^{-\int_{v}^{s} h(u) d u}\left(\sum_{j=1}^{n}\left|c_{j}(v)\right| \cdot\left|x\left(v-\delta_{j}(v)\right)-y\left(v-\delta_{j}(v)\right)\right|\right)^{2} d v\right\} \\
& \leq e \sup _{s \geq m\left(t_{0}\right)}|x(s)-y(s)|^{2} \\
& \cdot \sup _{s \geq t_{0}}\left\{( 1 + \frac { 1 } { l } ) \left(\sum_{j=1}^{n}\left|q_{j}(s)\right|+\sum_{j=1}^{n} \int_{t_{0}}^{s} e^{-\int_{v}^{s} h(u) d u}|h(v)| \int_{v-\tau_{j}(v)}^{v}\left|h_{j}(u)\right| d u d s\right.\right. \\
& +\sum_{j=1}^{n} \int_{s-\tau_{j}(s)}^{s}\left|h_{j}(v)\right| d v \\
& +\sum_{j=1}^{n} \int_{t_{0}}^{s} e^{-\int_{v}^{s} h(u) d u} \\
& \times \mid\left(h_{j}\left(v-\tau_{j}(v)\right)\left(1-\tau_{j}^{\prime}(v)\right)+b_{j}(v)-q_{j}(v) h(v) \mid d v\right)^{2} \\
& \left.+4(1+l) \int_{t_{0}}^{s} e^{-2 \int_{v}^{s} h(u) d u}\left(\sum_{j=1}^{n}\left|c_{j}(v)\right|\right)^{2} d v\right\} \leq\left(\alpha^{2}+\varepsilon\right) e \sup _{s \geq m\left(t_{0}\right)}|x(s)-y(s)|^{2} .
\end{aligned}
$$


Therefore, $P$ is contraction mapping with contraction constant $\alpha^{2}+\varepsilon$. By the contraction mapping principle, $P$ has a fixed point $x \in S$, which is a solution of (2.7) with $x(s)=\phi(s)$ on $\left[m\left(t_{0}\right), t_{0}\right]$ and $E|x(t)|^{2} \rightarrow 0$ as $t \rightarrow \infty$.

To obtain the mean square asymptotic stability, we need to show that the zero solution of (2.7) is mean square stable. Let $\epsilon>0$ be given and choose $\delta>0$ and $\delta<\epsilon$ satisfying the following condition:

$$
4 \delta K^{2}(1+L) e^{2 \int_{0}^{t_{0}} H(u) d u}+\left(\alpha^{2}+\varepsilon\right) \epsilon<\epsilon,
$$

where $K=\sup _{t>0}\left\{e^{-\int_{0}^{t} H(s) d s}\right\}$. If $x(t)=x\left(t, t_{0}, \phi\right)$ is a solution of (2.7) with $\|\phi\|^{2}<\delta$, then $x(t)=(P x)(t)$ defined in (2.13). We assume that $E|x(t)|^{2}<\epsilon$ for all $t \geq t_{0}$. Notice that $E|x(t)|^{2}=$ $\|\phi\|^{2}<\epsilon$ for $t \in\left[m\left(t_{0}\right), t_{0}\right]$. If there exists $t^{*}>t_{0}$ such that $E\left|x\left(t^{*}\right)\right|^{2}=\epsilon$ and $E|x(t)|^{2}<\epsilon$ for $t \in\left[m\left(t_{0}\right), t^{*}\right)$, then (2.13) and (2.19) imply that

$$
\begin{aligned}
& E\left|x\left(t^{*}\right)\right|^{2} \leq(1+L)\|\phi\|^{2}\left(1+\sum_{j=1}^{n}\left|q_{j}\left(t_{0}\right)\right|+\sum_{j=1}^{n} \int_{t_{0}-\tau_{j}\left(t_{0}\right)}^{t_{0}}\left|h_{j}(s)\right| d s\right)^{2} e^{-2 \int_{t_{0}}^{t^{*}} H(u) d u} \\
& +\epsilon\left(1+\frac{1}{L}\right)\left(\sum_{j=1}^{n}\left|q_{j}\left(t^{*}\right)\right|+\sum_{j=1}^{n} \int_{t^{*}-\tau_{j}\left(t^{*}\right)}^{t^{*}}\left|h_{j}(s)\right| d s\right.
\end{aligned}
$$

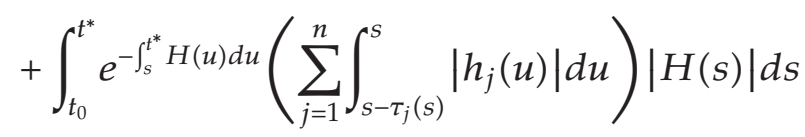

$$
\begin{aligned}
& \left.+\int_{t_{0}}^{t^{*}} e^{-\int_{s}^{t^{*}} H(u) d u} \sum_{j=1}^{n}\left|h_{j}\left(s-\tau_{j}(s)\right)\left(1-\tau_{j}^{\prime}(s)\right)+b_{j}(s)-q_{j}(s) H(s)\right| d s\right)^{2} \\
& +\epsilon \int_{t_{0}}^{t^{*}} e^{-2 \int_{s}^{t^{*}} H(u) d u}\left(\sum_{j=1}^{n}\left|c_{j}(s)\right|\right)^{2} d s \\
& \leq(1+L) \delta\left(1+\sum_{j=1}^{n}\left|q_{j}\left(t_{0}\right)\right|+\sum_{j=1}^{n} \int_{t_{0}-\tau_{j}\left(t_{0}\right)}^{t_{0}}\left|h_{j}(s)\right| d s\right)^{2} e^{-2 \int_{t_{0}}^{t^{*}} H(u) d u}+\left(\alpha^{2}+\varepsilon\right) \epsilon<\epsilon,
\end{aligned}
$$

which contradicts the definition of $t^{*}$. Thus, the zero solution of (2.7) is stable. It follows that the zero solution of (2.7) is mean square asymptotically stable if (2.11) holds.

Conversely, we suppose that (2.11) fails. From (i), there exists a sequence $\left\{t_{n}\right\}$ with $t_{n} \rightarrow \infty$ as $n \rightarrow \infty$ such that $\lim _{n \rightarrow \infty} \int_{0}^{t_{n}} H(u) d u=\beta$, where $\beta \in \mathbb{R}$. Then, we can choose a constant $J>0$ satisfying $\int_{0}^{t_{n}} H(u) d u \in[-J, J]$ for all $n \geq 1$. Denote

$$
\omega(s)=\sum_{j=1}^{n} \mid\left(h_{j}\left(s-\tau_{j}(s)\right)\left(1-\tau_{j}^{\prime}(s)\right)+b_{j}(s)-q_{j}(s) H(s)|+| H(s)\left|\int_{s-\tau_{j}(s)}^{s}\right| h_{j}(u) \mid d u\right.
$$

for all $s \geq 0$. From (ii), we have

$$
\int_{0}^{t_{n}} e^{-\int_{s}^{t_{n}} H(u) d u} \omega(s) d s \leq \alpha,
$$


which implies

$$
\int_{0}^{t_{n}} e^{\int_{0}^{s} H(u) d u} \omega(s) d s \leq \alpha e^{\int_{0}^{t_{n}} H(u) d u} \leq e^{J}
$$

Therefore, the sequence $\left\{\int_{0}^{t_{n}} e^{\int_{0}^{s} H(u) d u} \omega(s) d s\right\}$ has a convergent subsequence. Without loss of generality, we can assume that

$$
\lim _{n \rightarrow \infty} \int_{0}^{t_{n}} e^{\int_{0}^{s} H(u) d u} \omega(s) d s=\gamma
$$

for some $\gamma>0$. Let $k$ be an integer such that

$$
\int_{t_{k}}^{t_{n}} e^{\int_{0}^{s} H(u) d u} \omega(s) d s<\frac{\delta_{0}}{8 K}
$$

for all $n \geq k$, where $\delta_{0}>0$ satisfies $8 \delta_{0} K^{2} e^{2 J}+\left(\alpha^{2}+\varepsilon\right)<1$.

Now we consider the solution $x(t)=x\left(t, t_{k}, \phi\right)$ of $(2.7)$ with $\left\|\phi\left(t_{k}\right)\right\|^{2}=\delta_{0}$ and $\|\phi(s)\|^{2}<$ $\delta_{0}$ for $s<t_{k}$. By the similar method in (2.22), we have $E|x(t)|^{2}<1$ for $t \geq t_{k}$. We may choose $\phi$ so that

$$
G\left(t_{k}\right):=\phi\left(t_{k}\right)-\sum_{j=1}^{n} q_{j}\left(t_{k}\right) \phi\left(t_{k}-\tau_{j}\left(t_{k}\right)\right)-\sum_{j=1}^{n} \int_{t_{k}-\tau_{j}\left(t_{k}\right)}^{t_{k}} h_{j}(s) \phi(s) d s \geq \frac{1}{2} \delta_{0} .
$$

It follows from (2.13) and (2.28) with $x(t)=(P x)(t)$ that for $n \geq k$,

$$
\begin{gathered}
E\left|x\left(t_{n}\right)-\sum_{j=1}^{n} q_{j}\left(t_{n}\right) x\left(t_{n}-\tau_{j}\left(t_{n}\right)\right)-\sum_{j=1}^{n} \int_{t_{n}-\tau_{j}\left(t_{n}\right)}^{t_{n}} h_{j}(s) x(s) d s\right|^{2} \\
\geq G^{2}\left(t_{k}\right) e^{-2 \int_{t_{k}}^{t_{n}} H(u) d u}-2 G\left(t_{k}\right) e^{-\int_{t_{k}}^{t_{n}} H(u) d u} \int_{t_{k}}^{t_{n}} e^{-t_{s}^{t_{n}} H(u) d u} \omega(s) d s \\
\geq \frac{\delta_{0}}{2} e^{-2 \int_{t_{k}}^{t_{n}} H(u) d u}\left(\frac{\delta_{0}}{2}-2 K \int_{t_{k}}^{t_{n}} e^{\int_{0}^{s} H(u) d u} \omega(s) d s\right) \geq \frac{\delta_{0}^{2}}{8} e^{-2 J}>0 .
\end{gathered}
$$

If the zero solution of (2.7) is mean square asymptotic stable, then $E|x(t)|^{2}=$ $E\left|x\left(t, t_{k}, \phi\right)\right|^{2} \rightarrow 0$ as $t \rightarrow 0$. Since $t_{n}-\tau_{j}\left(t_{n}\right) \rightarrow \infty, t_{n}-\delta_{j}\left(t_{n}\right) \rightarrow \infty$ as $n \rightarrow \infty$ and condition (ii) and (2.11) hold,

$$
E\left|x\left(t_{n}\right)-\sum_{j=1}^{n} q_{j}\left(t_{n}\right) x\left(t_{n}-\tau_{j}\left(t_{n}\right)\right)-\sum_{j=1}^{n} \int_{t_{n}-\tau_{j}\left(t_{n}\right)}^{t_{n}} h_{j}(s) x(s) d s\right|^{2} \longrightarrow 0, \quad \text { as } n \longrightarrow \infty,
$$

which contradicts (2.29). Therefore, (2.11) is necessary for Theorem 2.1. This completes the proof.

Remark 2.2. Theorem 2.1 still holds if condition (ii) is satisfied for $t \geq t_{a}$ for some $t_{a} \in \mathbb{R}^{+}$. 
Remark 2.3. Theorem 2.1 improves Theorem C under different conditions.

Corollary 2.4. Suppose that $\tau_{j}$ is differential, the inverse function $g_{j}(t)$ of $t-\tau_{j}(t)$ exists, and there exists a constant $\alpha \in(0,1)$ such that for $t \geq 0, \liminf _{t \rightarrow \infty} \int_{0}^{t} Q(s) d s>-\infty$ and

$$
\begin{aligned}
& \sum_{j=1}^{n}\left|q_{j}(t)\right|+\sum_{j=1}^{n} \int_{t-\tau_{j}(t)}^{t}\left|b_{j}\left(g_{j}(s)\right)\right| d s+\sum_{j=1}^{n} \int_{0}^{t} e^{-\int_{s}^{t} Q(u) d u}\left|b_{j}(s) \tau_{j}^{\prime}(s)-q_{j}(s) Q(s)\right| d s
\end{aligned}
$$

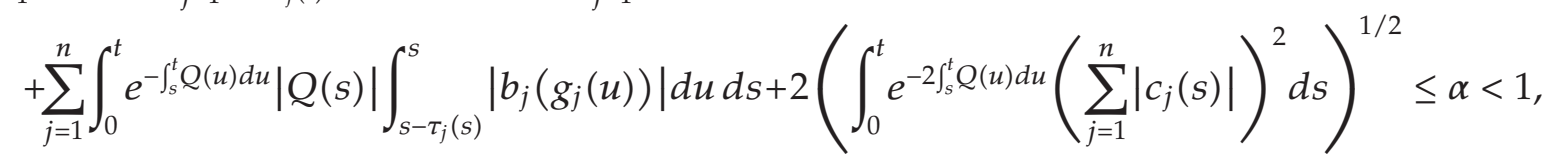

where $Q(t)=\sum_{j=1}^{n}-b_{j}\left(g_{j}(t)\right)$. Then the zero solution of $(2.7)$ is mean square asymptotically stable if and only if $\int_{0}^{t} Q(s) d s \rightarrow \infty$ as $t \rightarrow \infty$.

Remark 2.5. When $h_{j}(t)=-b_{j}\left(g_{j}(t)\right)$ for $j=1 \cdots n$, Theorem 2.1 reduces to Corollary 2.4. On the other hand, we choose $q_{j}(t) \equiv c_{j}(t) \equiv 0$ and $b_{j} \equiv-\bar{b}_{j}$ for $j=1 \cdots n$, then Corollary 2.4 reduces to Theorem B.

\section{Two examples}

In this section, we give two examples to illustrate applications of Theorem 2.1 and Corollary 2.4 .

Example 3.1. Consider the following linear neutral stochastic delay differential equation:

$$
d\left(x(t)-\frac{x(t-t / 2)}{1000}\right)=\left(-\frac{x(t-t / 2)}{16+16 t}-\frac{3 \sin t+4}{48+48 t} x\left(t-\frac{t}{4}\right)\right) d t+\left(\frac{x(t)}{24 \sqrt{3+4 t}}-\frac{x(t-\sin t)}{12 \sqrt{3+4 t}}\right) d W(t) .
$$

Then the zero solution of (3.1) is mean square asymptotically stable.

Proof. Choosing $h_{1}(t)=1 /(8+16 t)$ and $h_{2}(t)=7 /(48+64 t)$ in Theorem 2.1, we have

$$
\begin{aligned}
& H(t)=\frac{1}{8+16 t}+\frac{7}{48+64 t}, \quad \frac{11}{48+64 t} \leq H(t) \leq \frac{13}{48+64 t}, \\
& \sum_{j=1}^{2} \int_{t-\tau_{j}(t)}^{t}\left|h_{j}(s)\right| d s=\int_{t / 2}^{t} \frac{1}{8+16 s} d s+\int_{3 t / 4}^{t} \frac{7}{48+64 s} d s \longrightarrow 0.07479, \quad \text { as } t \longrightarrow \infty, \\
& \sum_{j=1}^{2} \int_{0}^{t} e^{-\int_{s}^{t} H(u) d u}|H(s)| \int_{s-\tau_{j}(s)}^{s}\left|h_{j}(u)\right| d u d s \leq \int_{0}^{t} e^{-\int_{s}^{t}(11 /(48+64 u)) d u} \frac{13}{48+64 s} \cdot 0.07479 d s \leq 0.08839, \\
& 2\left(\int_{0}^{t} e^{-2 \int_{s}^{t} H(u) d u}\left(\sum_{j=1}^{2}\left|c_{j}(s)\right|\right)^{2} d s\right)^{1 / 2} \leq 2\left(\int_{0}^{t} e^{-\int_{s}^{t}(11 /(24+32 u)) d u} \frac{1}{8(24+32 s)} d s\right)^{1 / 2} \leq 0.21320, \\
& \sum_{j=1}^{2} \int_{0}^{t} e^{-\int_{s}^{t} H(u) d u} \mid\left(h_{j}\left(s-\tau_{j}(s)\right)\left(1-\tau_{j}^{\prime}(s)\right)+b_{j}(s)-q_{j}(s) H(s) \mid d s\right. \\
& \leq \int_{0}^{t} e^{-\int_{s}^{t}(11 /(48+64 u)) d u}\left(\frac{0.013}{48+64 s}+\frac{17}{144+192 s}\right) d s \leq \frac{0.013}{11}+\frac{17}{33}=0.51634 .
\end{aligned}
$$


It easy to check that $\int_{0}^{\infty} H(s) d s=\infty$. Let $\alpha=0.001+0.07479+0.08839+0.21320+0.51634$. Then, $\alpha=0.89372<1$ and the zero solution of (3.1) is mean square asymptotically stable by Theorem 2.1.

Example 3.2. Consider the following delay differential equation:

$$
x^{\prime}(t)=-\frac{1}{6+4 t} x\left(t-\frac{t}{3}\right)-\frac{1}{12+4 t} x\left(t-\frac{2}{3} t\right) .
$$

Then the zero solution of (3.3) is asymptotically stable.

Proof. Choosing $h_{1}(t)=h_{2}(t)=1 /(4+4 t)$ in Theorem 2.1, we have $H(t)=1 /(2+2 t)$ and

$$
\begin{aligned}
& \sum_{j=1}^{2} \int_{t-\tau_{j}(t)}^{t}\left|h_{j}(s)\right| d s=\int_{(2 / 3) t}^{t} \frac{1}{4+4 s} d s+\int_{t / 3}^{t} \frac{1}{4+4 s} d s \rightarrow \frac{1}{2} \ln 3-\frac{1}{4} \ln 2=0.37602, \quad \text { as } t \longrightarrow \infty, \\
& \sum_{j=1}^{2} \int_{0}^{t} e^{-\int_{s}^{t} H(u) d u}|H(s)| \int_{s-\tau_{j}(s)}^{s}\left|h_{j}(u)\right| d u d s \leq \int_{0}^{t} e^{-\int_{s}^{t}(1 /(2+2 u)) d u} \frac{1}{2+2 s} \cdot 0.37602 d s \leq 0.37602 .
\end{aligned}
$$

Notice that $q_{j}(t)=c_{j}(t) \equiv 0$ and

$$
\begin{aligned}
& \sum_{j=1}^{2} \mid\left(h_{j}\left(s-\tau_{j}(s)\right)\left(1-\tau_{j}^{\prime}(s)\right)+b_{j}(s)-q_{j}(s) H(s) \mid\right. \\
& \quad=\left|\frac{3}{12+8 s} \cdot \frac{2}{3}-\frac{1}{6+4 s}\right|+\left|\frac{3}{12+4 s} \cdot \frac{1}{3}-\frac{1}{12+4 s}\right|=0 .
\end{aligned}
$$

It is easy to see that all the conditions of Theorem 2.1 hold for $\alpha=0.37602+0.37602=0.75204<$ 1. Thus, Theorem 2.1 implies that the zero solution of (3.3) is asymptotically stable.

However, Theorem B cannot be used to verify that the zero solution of (3.3) is asymptotically stable. In fact, $\bar{b}_{1}(t)=1 /(6+4 t), \bar{b}_{2}(t)=1 /(12+4 t), \bar{b}_{1}\left(g_{1}(t)\right)=1 /(6+6 t)$, $\bar{b}_{2}\left(g_{2}(t)\right)=1 /(12+12 t)$, and $|Q(t)|=1 /(4+4 t)$. As $t \rightarrow \infty$,

$$
\sum_{j=1}^{2} \int_{t-\tau_{j}(t)}^{t}\left|\bar{b}_{j}\left(g_{j}(s)\right)\right| d s \leq \int_{(2 / 3) t}^{t} \frac{1}{6+6 s} d s+\int_{t / 3}^{t} \frac{1}{12+12 s} d s \rightarrow \frac{1}{4} \ln 3-\frac{1}{6} \ln 2=0.15913 .
$$

Notice that

$$
\sum_{j=1}^{2}\left|\bar{b}_{j}(s) \tau_{j}^{\prime}(s)-q_{j}(s) Q(s)\right|=\frac{1}{18+12 s}+\frac{1}{18+6 s} \leq \frac{1}{4+4 s} .
$$

It follows from (3.7) that

$$
\sum_{j=1}^{2} \int_{0}^{t} e^{-\int_{s}^{t} Q(u) d u}\left|\bar{b}_{j}(s) \tau_{j}^{\prime}(s)-q_{j}(s) Q(s)\right| d s \leq \int_{0}^{t} e^{-\int_{s}^{t}(1 /(4+4 u)) d u} \frac{1}{4+4 s} d s \leq 1 .
$$


From (3.6), we obtain

$$
\sum_{j=1}^{2} \int_{0}^{t} e^{-\int_{s}^{t} Q(u) d u}|Q(s)| \int_{s-\tau_{j}(s)}^{s}\left|\bar{b}_{j}\left(g_{j}(u)\right)\right| d u d s \leq \int_{0}^{t} e^{-\int_{s}^{t}(1 /(4+4 u)) d u} \frac{1}{4+4 s} \cdot 0.15913 d s \leq 0.15913 .
$$

Combining (3.6), (3.8), and (3.9), we see that the condition (2.4) of Theorem B does not hold with $\alpha=1.31825$.

\section{Acknowledgement}

This work was supported by the National Natural Science Foundation of China (10671135) and Specialized Research Fund for the Doctoral Program of Higher Education (20060610005).

\section{References}

[1] T. A. Burton, "Stability by fixed point theory or Liapunov theory: a comparison," Fixed Point Theory, vol. 4, no. 1, pp. 15-32, 2003.

[2] T. A. Burton, "Liapunov functionals, fixed points, and stability by Krasnoselskii's theorem," Nonlinear Studies, vol. 9, no. 2, pp. 181-190, 2002.

[3] T. A. Burton, "Fixed points and stability of a nonconvolution equation," Proceedings of the American Mathematical Society, vol. 132, no. 12, pp. 3679-3687, 2004.

[4] T. A. Burton and T. Furumochi, "Fixed points and problems in stability theory for ordinary and functional differential equations," Dynamic Systems and Applications, vol. 10, no. 1, pp. 89-116, 2001.

[5] S.-M. Jung, "A fixed point approach to the stability of a Volterra integral equation," Fixed Point Theory and Applications, vol. 2007, Article ID 57064, 9 pages, 2007.

[6] J. Luo, "Fixed points and stability of neutral stochastic delay differential equations," Journal of Mathematical Analysis and Applications, vol. 334, no. 1, pp. 431-440, 2007.

[7] B. Zhang, "Fixed points and stability in differential equations with variable delays," Nonlinear Analysis, vol. 63, no. 5-7, pp. e233-e242, 2005.

[8] V. B. Kolmanovskii and L. E. Shaikhet, "Matrix Riccati equations and stability of stochastic linear systems with nonincreasing delays," Functional Differential Equations, vol. 4, no. 3-4, pp. 279-293, 1997.

[9] K. Liu, Stability of Infinite Dimensional Stochastic Differential Equation with Applications, vol. 135 of Chapman \& Hall/CRC Monographs and Surveys in Pure and Applied Mathematics, Chapman \& Hall/CRC, Boca Raton, Fla, USA, 2006.

[10] I. Karatzas and S. E. Shreve, Brownian Motion and Stochastic Calculus, vol. 113 of Graduate Texts in Mathematics, Springer, New York, NY, USA, 2nd edition, 1991. 